Published in final edited form as:

Cancer Causes Control. 2019 February ; 30(2): 165-168. doi:10.1007/s10552-018-1095-z.

\title{
Non-alcoholic fatty liver disease and colorectal cancer survival
}

\author{
Kana Wu${ }^{1}$, Mike Z. Zhai ${ }^{2}$, Erin K. Weltzien ${ }^{3}$, Elizabeth M. Cespedes Feliciano ${ }^{3}$, Jeffrey A. \\ Meyerhardt $^{4}$, Edward Giovannucci ${ }^{1,5}$, and Bette J. Caan ${ }^{3}$ \\ ${ }^{1}$ Department of Nutrition, Harvard T.H. Chan School of Public Health, Boston, MA, USA \\ ${ }^{2}$ Harvard Medical School, Boston, MA, USA \\ ${ }^{3}$ Division of Research, Kaiser Permanente Northern California, Oakland, CA, USA \\ ${ }^{4}$ Department of Medical Oncology, Dana-Farber Cancer Institute, Harvard Medical School, \\ Boston, MA, USA \\ ${ }^{5}$ Department of Epidemiology, Harvard T.H. Chan School of Public Health, Boston, MA, USA
}

\begin{abstract}
Purpose-Liver diseases including non-alcoholic fatty liver disease (NAFLD) and ensuing alterations to the micro-environment may affect development of liver metastasis. Mirroring the rise in obesity rates, prevalence of NAFLD is increasing globally. Our objective was to examine the association between NAFLD and mortality in colorectal cancer patients.
\end{abstract}

Methods-Colorectal Cancer-Sarcopenia and Near-term Survival (C-SCANS) is a retrospective cohort study which included 3,262 stage I-III patients, aged 18-80 years, and diagnosed between 2006 and 2011 at Kaiser Permanente Northern California. Cox proportional hazards regression was used to calculate multivariable adjusted hazard ratios (HR) and 95\% confidence intervals (CI).

Results-After up to 10 years of follow-up, 879 deaths, including 451 from CRC were identified. Cases diagnosed with NAFLD before and within 1 month after CRC diagnosis (preexisting NAFLD; $n=83$ ) had a HR of 1.64 (95\% CI 1.06-2.54) for overall and a HR of 1.85 (95\% CI 1.03-3.30) for CRC-specific mortality compared to those without NAFLD. Findings did not differ significantly by sex, stage, tumor location, and smoking status, and were also similar when restricted to obese patients only.

Conclusions-Independent of body mass index and prognostic indicators, CRC patients with pre-existing NAFLD had a worse prognosis than those without NAFLD.

\section{Keywords}

Non-alcoholic fatty liver disease; Colorectal cancer survival; Liver metastasis

Kana Wu KWU@hsph.harvard.edu.

Electronic supplementary material The online version of this article (https://doi.org/10.1007/s10552-018-1095-z) contains supplementary material, which is available to authorized users. 


\section{Introduction}

Colorectal cancer (CRC) is the third most common cancer worldwide and the fourth common cause of cancer death worldwide [1]. In the US alone, 135, 430 new colorectal cases and 50,430 deaths due to colorectal cancer are expected in 2017 [2]. Despite "curative" surgical resection, over $60 \%$ of stage I-III CRC patients will eventually relapse or develop distant metastases, mainly in the liver. Development of liver metastasis among stage I-III CRC patients after resection suggests the presence of micro-metastases prior to the diagnosis of CRC [3].

Although several decades ago scientists suggested that pre-existing liver diseases including fatty liver and ensuing alterations to the micro-environment may affect development of liver metastasis, only few epidemiological studies have pursued this hypothesis [4-8]. Mirroring the recent rise in obesity and type II diabetes rates, non-alcoholic fatty liver disease (NAFLD) and its more severe form, non-alcoholic steatohepatitis (NASH), are quickly emerging as the most common liver diseases with an estimated prevalence of NAFLD of about $25 \%$ worldwide $[9,10]$.

The objective of this study was to examine the association between pre-existing NAFLD and overall and CRC-specific mortality in stage I-III CRC patients utilizing data from the CSCANS (Colorectal Cancer-Sarcopenia and Near-term Survival) project.

\section{Methods}

The Colorectal Cancer-Sarcopenia and Near-term Survival (C-SCANS) is a retrospective cohort study, which included 3,262 patients at Kaiser Permanente Northern California, aged 18-80 years, who were diagnosed with stage I-III colorectal cancer and underwent surgical resection between 2006 and 2011 [11, 12]. More detail about this cohort can be found in our previous publications $[11,12]$.

All-cause deaths and CRC deaths were identified from KPNC mortality files which encompasses data from the California State Department of Vital Statistics, U.S. Social Security Administration, and KPNC utilization data sources. Death certificates were reviewed to confirm all-cause and cause-specific deaths. CRC-specific deaths are defined as those where CRC was the underlying or the contribution cause of death. Data on prognostic factors (stage, treatment data, primary tumor location, and histology) were extracted from electronic medical records at KPNC and the KPNC Cancer Registry. Information on sociodemographic variables, lifestyle (e.g., smoking status), height, and weight closest to diagnosis and before treatment was also obtained via linkage to KPNC electronic medical record data $[11,12]$.

Only patients with NAFLD diagnosed prior to or within 1 month after date of CRC diagnosis were defined as having "pre-existing" NAFLD based on International Classification of Diseases (ICD)-9 codes 571.8 or ICD-10-CM K76.0 and 75.81.

The study was approved by the KPNC Institutional Review Board. 


\section{Statistical analysis}

To investigate the association between NAFLD prior to CRC diagnosis and CRC and overall mortality, Cox proportional hazards regression models were used to calculate multivariable adjusted hazard ratios (HR) and 95\% confidence intervals (CI). Multivariable models included the following variables: age (continuous, years), sex, race/ethnicity (non-Hispanic white, black, Hispanic, or Asian/Pacific Islander), smoking (current, former, or never), tumor stage and grade, receipt of chemotherapy and/or radiation, cancer site (proximal, distal, or rectum) and body mass index (BMI, closest to diagnosis and before treatment, as continuous variable $\mathrm{kg} / \mathrm{m}^{2}$ ), and history of diabetes (yes, no). Participants contributed person time from the date of diagnosis of CRC to date of death (for overall survival), death with CRC being the primary or contributing cause, or end of follow-up, i.e., 30 December 2016. We also conducted analysis separately by sex, tumor stage (stage I/II vs. III), primary tumor location (proximal, distal, rectal), smoking status (never, past, current), receipt of chemotherapy or radiation treatment (yes vs. no), and BMI $(18-<25,25-<30$ and $\geq 30$ $\left.\mathrm{kg} / \mathrm{m}^{2}\right)$. A two-sided $p$ value of $<0.05$ was considered statistically significant.

\section{Results}

Among 3,262 patients, 879 deaths, including 451 from CRC, were identified. Median follow-up time was 6.6 years. NAFLD patients did not differ considerably from those without NAFLD by sex, stage, and smoking status. However, NAFLD patients with CRC were less likely to be non-Hispanic White, have received chemo- or radiation therapy, and were more likely obese and diagnosed with rectal and distal colon cancers and diabetes than those without NAFLD (Supplemental Material Table S1). According to the Kaplan-Meier survival function, patients with NAFLD ( $n=62 ; 33$ males, 29 females) had a shorter survival time than those without NAFLD (log-rank $p$ value $0.11 ;$ Fig. 1$)$. NAFLD patients had a multivariable adjusted HR of 1.64 (95\% CI 1.06-2.54) for overall and a multivariable adjusted HR of 1.84 (95\% CI 1.03-3.29) for CRC-specific mortality (Table 1). Results did not differ significantly after associations between NAFLD and total mortality were examined separately by sex, stage (I and II vs. III), location (proximal, distal, and rectal), smoking status (never, former, current), receipt of radiation, or chemotherapy (data not shown). Results for total mortality were also similar when we restricted analysis to CRC patients who were obese (BMI $\geq 30 \mathrm{~kg} / \mathrm{m}^{2}, 38$ NAFLD cases, and 14 deaths; HR 1.79, 95\% CI 1.03-3.11). We did not examine associations separately for normal weight and overweight patients because of small number of deaths among CRC patients with NAFLD $\left(18-<25 \mathrm{~kg} / \mathrm{m}^{2}: 11\right.$ NAFLD cases, 2 deaths and $25-<30 \mathrm{~kg} / \mathrm{m}^{2}, 13$ NAFLD cases, 5 deaths).

\section{Discussion}

In this retrospective cohort study, independent of BMI and other established prognostic indicators, CRC patients with pre-existing NAFLD had a worse prognosis than those without NAFLD prior to CRC diagnosis. Early autopsy studies from the US dating back to the 1940s have observed that cancer patients (multiple cancer sites) with liver cirrhosis were less likely to develop liver metastases than those without liver cirrhosis [4], suggesting that liver 
diseases and ensuing alterations to the liver microenvironment may affect development of liver metastasis ("seed-soil hypothesis" [13]). The liver is the most common site for CRC metastases [14], but only few observational studies, the majority conducted in Asian populations, have examined the association between pre-existing liver diseases and CRC liver metastasis or CRC prognosis. For example, a recent meta-analysis of 10 studies (9 Asian studies and one study from Italy) including 10, 349 CRC patients found that patients with chronic liver disease including hepatitis, cirrhosis, and fatty liver disease were less likely to develop liver metastasis (pooled OR 0.32, 95\% CI 0.26-0.38) [8]. However, in that meta-analysis only 3 studies examined associations for fatty liver disease and none specifically investigated NAFLD or NASH. Results from studies conducted after the publication of the aforementioned meta-analysis are conflicting. For example, one large Chinese study which included 1,314 CRC patients observed that patients with ultrasounddetected NAFLD had a longer overall survival but not disease-free survival [7]. In that study, inverse associations appeared to be restricted to patients with BMI ranging between 18.5 and $24.9 \mathrm{~kg} / \mathrm{m}^{2}$. Similarly, another study based in Japan which included 604 CRC patients, also reported that those with pre-existing hepatic steatosis [diagnosed via preoperative nonenhanced computed tomography (CT) scan] [15] exhibited a lower risk of developing liver metastasis when compared to those without hepatic steatosis.

Several mechanisms underlying NAFLD and its potential effect on development of liver metastasis have been proposed, though supporting data are limited. One animal study reported that in mice injected with colon cancer cells, those with steatotic livers developed more liver tumors than those without, suggesting that the micro-environment in fatty liver may facilitate growth of metastases [16].

Our study is the first study to investigate the association between NAFLD and CRC survival in a predominantly Caucasian population. Notably, we found that observed associations were independent of body mass index and were also similar when we restricted analysis to obese patients. However, one major limitation is that we identified NAFLD using ICD codes from EMRs, thus prevalence was underestimated. However, systematic bias is unlikely as only NAFLD cases diagnosed prior to CRC were included. Exposure misclassification should be non-differential and biases estimate towards null. Thus, actual associations may be even stronger than reported. Secondly, our outcome was mortality and we did not radiologically confirm liver metastasis. However, liver metastasis is the most common cause of CRC deaths [14]. Nonetheless, our findings warrant confirmation in future large-scale studies in CRC patients integrating clinical data and centralized review of CT scans to diagnose pre-existing NAFLD, which will also allow for a quantitative assessment of fat infiltration into the liver; and follow-up CT scans to confirm liver metastasis. Considering the rising prevalence of NAFLD worldwide, and that CRC is the fourth leading cause of cancer deaths globally, larger studies investigating the influence of NAFLD on liver microenvironment and metastasis are now urgently needed. Such studies will likely have major clinical implications, including optimizing treatment strategies and facilitating lifestyle interventions, risk stratification, and surveillance for stage I-III CRC patients. 


\title{
Supplementary Material
}

Refer to Web version on PubMed Central for supplementary material.

\section{Acknowledgments}

Funding Grant from the National Cancer Institute R01CA175011 to Dr. Bette Caan is acknowledged.

\author{
Abbreviations \\ BMI Body mass index \\ CRC Colorectal cancer \\ CI Confidence interval \\ HR Hazard ratio \\ KPNC Kaiser Permanente Northern California \\ NAFLD Non-alcoholic fatty liver disease
}

\section{References}

1. Arnold M, Sierra MS, Laversanne M, Soerjomataram I, Jemal A, Bray F (2017) Global patterns and trends in colorectal cancer incidence and mortality. Gut 66(4):683-691 [PubMed: 26818619]

2. Siegel RL, Miller KD, Fedewa SA, Ahnen DJ, Meester RGS, Barzi A, Jemal A (2017) Colorectal cancer statistics. Cancer J Clin 67(3):177-193

3. Andre N, Schmiegel W (2005) Chemoradiotherapy for colorectal cancer. Gut 54(8):1194-1202 [PubMed: 16009693]

4. Lieber MM (1957) The rare occurrence of metastatic carcinoma in the cirrhotic liver. Am J Med Sci 233(2):145-152 [PubMed: 13394591]

5. Le MH, Devaki P, Ha NB, Jun DW, Te HS, Cheung RC, Nguyen MH (2017) Prevalence of nonalcoholic fatty liver disease and risk factors for advanced fibrosis and mortality in the United States. PLoS ONE 12(3):e0173499

6. Min YW, Yun HS, Chang WI, Kim JY, Kim YH, Son HJ, Kim JJ, Rhee JC, Chang DK (2012) Influence of non-alcoholic fatty liver disease on the prognosis in patients with colorectal cancer. Clin Res Hepatol Gastroenterol 36(1):78-83 [PubMed: 22133576]

7. You J, Huang S, Huang GQ, Zhu GQ, Ma RM, Liu WY, Shi KQ, Guo GL, Chen YP, Braddock M, Zheng MH (2015) Nonalcoholic fatty liver disease: a negative risk factor for colorectal cancer prognosis. Medicine 94(5):e479

8. Cai B, Liao K, Song XQ, Wei WY, Zhuang Y, Zhang S (2014) Patients with chronically diseased livers have lower incidence of colorectal liver metastases: a meta-analysis. PLoS ONE 9(9):e108618

9. Younossi ZM, Koenig AB, Abdelatif D, Fazel Y, Henry L, Wymer M (2016) Global epidemiology of nonalcoholic fatty liver dis-ease-meta-analytic assessment of prevalence, incidence, and outcomes. Hepatology 64(1):73-84 [PubMed: 26707365]

10. Rinella M, Charlton M (2016) The globalization of nonalcoholic fatty liver disease: prevalence and impact on world health. Hepatology 64(1):19-22 [PubMed: 26926530]

11. Cespedes Feliciano EM, Kroenke CH, Meyerhardt JA, Prado CM, Bradshaw PT, Dannenberg AJ, Kwan ML, Xiao J, Quesenberry C, Weltzien EK, Castillo AL, Caan BJ (2016) Metabolic dysfunction, obesity, and survival among patients with early-stage colorectal cancer. J Clin Oncol 30:3664

12. Meyerhardt JA, Kroenke CH, Prado CM, Kwan ML, Castillo A, Weltzien E, Cespedes Feliciano EM, Xiao J, Caan BJ (2017) Association of weight change after colorectal cancer diagnosis and 
outcomes in the Kaiser Permanente Northern California Population. Cancer Epidemiol Biomark Prev 26(1):30-37

13. Langley RR, Fidler IJ (2011) The seed and soil hypothesis revisited-the role of tumor-stroma interactions in metastasis to different organs. Int J Cancer 128(11):2527-2535 [PubMed: 21365651]

14. Stangl R, Altendorf-Hofmann A, Charnley RM, Scheele J (1994) Factors influencing the natural history of colorectal liver metastases. Lancet 343(8910):1405-1410 [PubMed: 7515134]

15. Murono K, Kitayama J, Tsuno NH, Nozawa H, Kawai K, Sunami E, Akahane M, Watanabe T (2013) Hepatic steatosis Is associated with lower incidence of liver metastasis from colorectal cancer. Int J Colorectal Dis 28(8):1065-1072 [PubMed: 23392476]

16. VanSaun MN, Lee IK, Washington MK, Matrisian L, Gorden DL (2009) High fat diet induced hepatic steatosis establishes a permissive microenvironment for colorectal metastases and promotes primary dysplasia in a murine model. Am J Pathol 175(1):355-364 [PubMed: 19541928] 
log-rank p-value 0.11

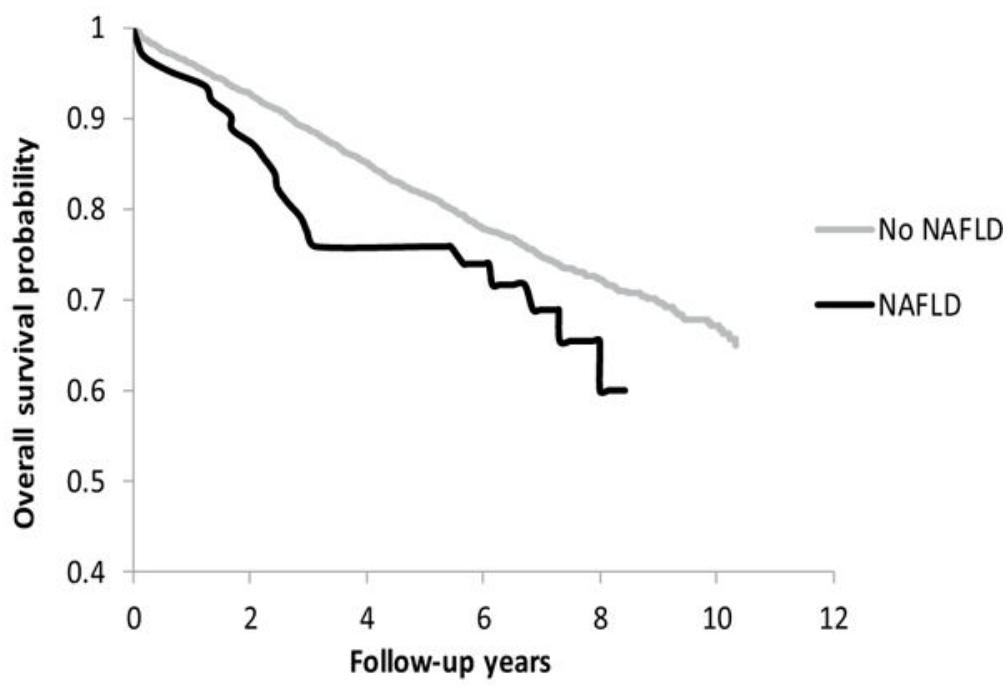

\#At risk:

No NAFLD

$3200 \quad 2968$

$2723 \quad 2014$

1032

1730

$62 \quad 55$

$\begin{array}{lllll}47 & 34 & 11 & 1 & 0\end{array}$

Fig. 1.

Kaplan-Meier curves for overall mortality 


\section{Table 1}

Associations between pre-CRC diagnosis of NAFLD and overall as well as CRC-specific mortality

\begin{tabular}{|c|c|c|c|}
\hline & Patients no. & Events no. & $\begin{array}{l}\text { NAFLD } \\
\operatorname{HR}^{a}(95 \% \mathrm{CI})\end{array}$ \\
\hline \multicolumn{4}{|c|}{ Overall mortality } \\
\hline No NAFLD & 3,200 & 858 & 1.00 [reference] \\
\hline NAFLD & 62 & 21 & $1.64(1.06-2.54)$ \\
\hline \multicolumn{4}{|c|}{ CRC-specific mortality ${ }^{b}$} \\
\hline No NAFLD & 2,987 & 439 & 1.00 [reference] \\
\hline NAFLD & 57 & 12 & $1.84(1.03-3.29)$ \\
\hline
\end{tabular}

${ }^{a}$ Cox regression models adjusted for sex (male vs. female), age at diagnosis (continuous, years), race (Whites, Blacks, Hispanic, or Asian vs. nonHispanic White), tumor stage (I or II vs. III), receipt of chemotherapy and/or radiation (not received vs. received), cancer site (proximal, distal, rectal), smoking history (current or former vs. never), and history of diabetes, and body mass index (as continuous variable in $\mathrm{kg} / \mathrm{m}^{2}$ )

${ }^{b}$ Cause of death is missing, thus the total number of patients does not add up to 3,262 